\title{
Granulicatella adiacens endocarditis of a complex congenital heart disease on an adult
}

\author{
Nilda Espinola-Zavaleta ${ }^{1,2, a^{*}}$, Luis Javier Castellanos-Vizcaíno ${ }^{1,3, a}$, Andrea Salazar-Vitale ${ }^{3}$, Rodrigo Escalante-Armenta ${ }^{1,4}$ and Erick \\ Alexanderson-Rosas ${ }^{1,5}$ \\ ${ }^{1}$ Department of Nuclear Cardiology, National Institute of Cardiology Ignacio Chávez, Mexico City, Mexico \\ ${ }^{2}$ Department of Echocardiography, ABC Medical Center I.A.P., Mexico City, Mexico \\ ${ }^{3}$ Faculty of Health Sciences, Anahuac University, State of Mexico, Mexico \\ ${ }^{4}$ Mexican Faculty of Medicine, La Salle University, Mexico City, Mexico \\ ${ }^{5}$ Physiology Department, National Autonomous University of Mexico, Mexico City, Mexico \\ ${ }^{\mathrm{a} E q u a l}$ contribution
}

\begin{abstract}
We present a 23-year-old man with complex CHD, who came to our institution due to moderate exertion dyspnea and fever. He was diagnosed with mitral valve endocarditis and Granulicatella adiacens was isolated. He was treated with antibiotics and discharged as he refused surgery. He came back on August 2019 with progressive increase of abdominal perimeter, lower limbs edema and chest pain. On cardiac examination pericardial rub and III/VI apical holosystolic murmur were found. The transthoracic echocardiogram showed situs solitus, atrioventricular discordance, double-outlet right ventricle with transposition of great arteries, interventricular septal defect, moderate tricuspid regurgitation, vegetations in both leaflets of mitral valve and severe mitral regurgitation, subvalvular pulmonary stenosis and huge pericardial effusion. The patient underwent successful mitral valve replacement and he was discharged in good condition.
\end{abstract}

\section{Introduction}

Atrioventricular discordance is a rare congenital heart disease, as it accounts for less than $1 \%$ of all of them [1]. It has been described that approximately $15 \%$ of the cases of atrioventricular discordance may also present with double outlet of the morphological right ventricle [2]. Several studies suggest a genetic predisposition as the main cause of congenital heart disease; the heterozygous ISL1 mutation (gene related with heart development transcription factors) was studied on 114 patients with congenital heart disease and compared with a control group of 218 subjects. It was found that ISL1 mutation was related to double outlet right ventricle (DORV) and ventricular septal defect [3]. Recently, the same clinical association has been found for the heterozygous MEF2C mutation [3].

Imaging techniques are crucial on the assessment of complex congenital heart disease, as it has implications on the surgical planning. Multislice computed tomography allows for the precise definition of the double outlet right ventricle's anatomy, as well as the ventricular septal defect and of the associated anomalies [4].

Granulicatella adiacens is a rare microorganism that has been isolated as the culprit of certain cases of infective endocarditis. Granulicatella adiacens is a nutritionally variant streptococci and may be found as commensal flora of the mouth, genital and intestinal tracts [5]. Granulicatella adiacens' infectious endocarditis tends to be underestimated, seeing as it is a microorganism with specific intrinsic features which make it hard to cultivate and isolate. For the same reason, the antimicrobial susceptibility data of this microorganism is not highly available. The antimicrobial susceptibility of Granulicatella adiacens was studied on six specimens and proved to have some important resistances: it two out of six isolates were resistant to penicillin; four out of six isolates were resistant to erythromycin and all six of them were resistant to both cefuroxime and ceftriaxone [6].

\section{Case presentation}

A 23-year-old man with no relevant family history, and an unknown and uncharacterized congenital heart disease only referred as "a patent ductus arteriosus" by the patient, who had been monitored in a third-level hospital.

He come to the emergency department of our institution with moderate exertion dyspnea, asthenia, fatigue, fever of $39^{\circ} \mathrm{C}$, hyporexia and the presence of bilateral lower limb's purpuric lesions.

On physical examination, grade III jugular ingurgitation, holosystolic murmur IV/VI in mesocardium and systolic murmur IV/ IV radiated to axilla, $3 \mathrm{~cm}$ hepatomegaly without ascites and lower limbs monomorphic dermatosis with petechiae were detected. Twoset blood culture was taken with isolation of Granulicatella adiacens. The echocardiogram showed situs solitus, atrioventricular discordance,

${ }^{\star}$ Correspondence to: Nilda Espinola-Zavaleta, MD, National Institute of Cardiology Ignacio Chavez, Juan Badiano No 1, Colonia Sección XVI, Tlalpan, P.C. 14080, Mexico City, Mexico, E-mail: niesza2001@hotmail.com

Key words: atrioventricular discordance, congenital heart disease, echocardiography, infectious endocarditis, granulicatella adiacens

Received: November 02, 2019; Accepted: November 14, 2019; Published: November 18, 2019 
double outlet right ventricle with transposition of the great vessels with pulmonary artery placed anteriorly and to the right and aorta in posterior position, perimembranous interventricular septal defect with bidirectional shunt, vegetations in both mitral leaflets and severe mitral regurgitation, mild tricuspid regurgitation and moderate subvalvular stenosis with peak gradient of $53 \mathrm{mmHg}$.

The patient was started on antibiotic regimen of ceftriaxone and amikacin. He was discharged after a 23-days hospitalization period, with diagnosis of infectious endocarditis and surgical indications, which he rejected due to personal issues. He was discharged with a 7-day regimen of amoxicillin/sulbactam.

The patient came back less than a month later with progressive increase of his abdominal perimeter, lower limbs edema dyspnea of mild efforts and chest pain. On physical examination, a pericardial rub and an IV/VI holosystolic mitral murmur were found. The abdominal examination revealed $2 \mathrm{~cm}$ hepatomegaly and secondgrade ascites.

After re-evaluation, it was concluded that the patient was a candidate for surgical intervention. A redirection of the systemic ventricle to the aorta with an intracardiac bovine pericardial patch tunnel, closure of the interventricular septal defect, Rastelli procedure with a $26 \mathrm{~mm}$ woven Dacron graft and a mitral valve replacement with a biological porcine St. Jude Epic $25 \mathrm{~mm}$ valve were performed. The patient evolved satisfactorily and was discharged in good condition after a 46 days-hospitalization (Figure 1).

\section{Discussion}

Atrioventricular discordance is a rare congenital cardiac malformation, which results from the abnormal rotation of the ventricles at the embryonic development of the heart causing an incorrect pairing of atria and ventricles [7]. This condition tends to be associated with double-outlet right ventricle and transposition of the great vessels when the ventricles undergo a counterclockwise rotation as is the case of the patient at issue [7]. Additionally, more than half of the patients who present this congenital defect also have ventricular septal defect [8]. Furthermore, of the few cases of atrioventricular discordance associated with DORV that have been described, pulmonary stenosis is a usual finding in these patients [7].

We present the case of a 23-year-old man with a complex congenital heart disease who was followed on a third level hospital for 15 years. Patients with complex congenital malformations have high mortality rates, and rarely reach adulthood [9], making this patient's condition extremely rare. In addition, the patient was asymptomatic until his second decade of life, which makes the case unusual due to the complexity of the malformation.

Moreover, cardiac malformations represent an important risk factor for developing infective endocarditis, and complicated conditions like our patient's precipitate even more the appearance of the disease. Granulicatella adiacens is part of the Nutritionally variant Streptococci (NVS) category, which are Gram positive bacteria belonging to the viridans group and are responsible for 5-6\% of all infective endocarditis' cases [9]. Infections from this microorganism

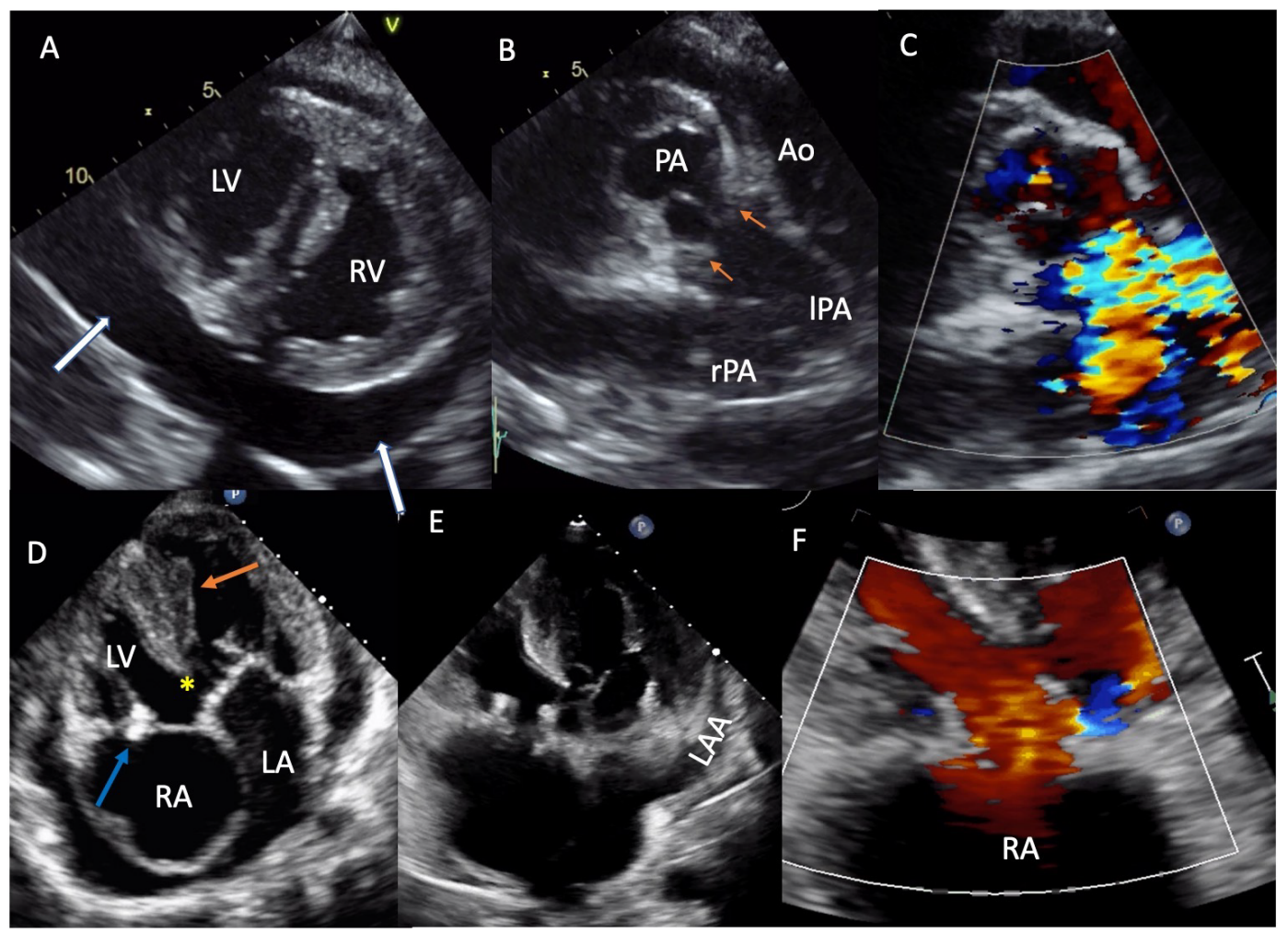

Figure 1. Transthoracic echocardiogram. A-Parasternal short axis view at the level of both ventricles with morphologically right ventricle to the left and morphologically left ventricle to the right and huge pericardial effusion (white arrows). B, C-Parasternal short axis view with transposition of great arteries with pulmonary artery placed anteriorly and to the right and aorta in posterior position and pulmonary subvalvular stenosis (orange arrows). D-Four chamber view with atrioventricular discordance, the orange arrow points the moderator band, the yellow asterisk shows the ventricular septal defect and the blue arrow points the mitral valve vegetations. E,F-Four chamber view $2 \mathrm{D}$ and color flow showing the normal functioning biological prosthetic mitral valve. Abbreviations: RA-right atrium; RV-right ventricle; LA-left atrium; LV-left ventricle; PA-pulmonary artery; 1PA-left pulmonary artery; rPA-right pulmonary artery; Ao-aorta; LAA-left atrial appendage 
tend to have high mortality rates, and usually have high levels of resistance to clindamycin, erythromycin, rifampicin and vancomycin [5].

\section{Conclusions}

These types of congenital cardiac malformations tend to be very rare and variable, and therefore should be evaluated individually from patient-to-patient. Proper management should, not only focus on correcting the malformation, but also on preventing and controlling any comorbidity that could arise, such the current case of infective endocarditis caused by a rare microorganism such as Granulicatella adiacens.

\section{Highlights}

- Atrioventricular discordance represents less than the $1 \%$ of congenital heart disease.

- Congenital heart diseases are an important risk factor for developing infective endocarditis.

Granulicatella adiacens is a rare cause of infective endocarditis that must be intentionally sought in the group of negative culture endocarditis.

\section{References}

1. Sharland G, Tingay R, Jones A, Simpson Je (2005) Atrioventricular and ventriculoarterial discordance (congenitally corrected transposition of the great arteries): echocardiographic features, associations, and outcome in 34 fetuses. Heart 91: $1453-1458$.

2. Battistessa S, Soto B (1990) Double outlet right ventricle with discordant atrioventricular connexion: an angiographic analysis of 19 cases. Int J Cardiol 27: 253-263.

3. Wang Z, Song HM, Wang F, Zhao CM, Huang RT, et al. (2019) A New ISL1 Loss-ofFunction Mutation Predisposes to Congenital Double Outlet Right Ventricle. Int Heart $J 60: 1113-1122$

4. Zheng X, Freeman AF, Villafranca J, Shortridge D, Beyer J, et al. (2004) Antimicrobia susceptibilities of invasive pediatric Abiotrophia and Granulicatella isolates. $J$ Clin Microbiol 42: 4323-4326.

5. Shailaja TS, Sathiavathy KA, Unni G (2013) Infective endocarditis caused by Granulicatella adiacens. Indian Heart J 65: 447-449.

6. Ren S, Ma C, Li S (2019) Criss-cross heart with double-outlet right ventricle, subpulmonary ventricular septal defect, and bicuspid pulmonary valve. J Clin Ultrasound 47: 315-318

7. Wilkinson J, Anderson R (2010) Anatomy of Discordant Atrioventricular Connections. World J Pediatr Congenit Heart Surg 2: 43-53.

8. Özkutlu S, Elshershari H, Akçören Z, Önderğolu L, Tekinalp G (2002) Visceroatrial situs solitus with atrioventricular alignment discordance double outlet right ventricle and superoinferior ventricles: Fetal and neonatal echocardiographic findings. $J$ Am Soc Echocardiogr 15: 749-752.

9. Padmaja K, Lakshmi V, Subramanian S, Neeraja M, Krishna S, et al. (2014) Infective endocarditis due to Granulicatella adiacens: a case report and review. J Infect Dev Ctries 8: 548-550.

Copyright: (C2019 Espinola-Zavaleta N. This is an open-access article distributed under the terms of the Creative Commons Attribution License, which permits unrestricted use, distribution, and reproduction in any medium, provided the original author and source are credited. 